\title{
GÉRARD-SIBUYA'S VERSUS MAJIMA'S CONCEPT OF ASYMPTOTIC EXPANSION IN SEVERAL VARIABLES
}

\author{
J. A. HERNÁNDEZ and J. SANZ \\ (Received 17 December 1999; revised 8 November 2000) \\ Communicated by P. Fenton
}

\begin{abstract}
We give an example of a holomorphic function, admitting Gérard-Sibuya asymptotic expansion on a polysector of $\mathbb{C}^{n}$, and such that none of its derivatives admits such an expansion. This motivates the study of the relationship between the concepts of asymptotic expansion in several variables respectively given by Gérard-Sibuya and Majima. For a function $f$, Majima's notion is proved to be equivalent, on the one hand, to the existence of Gérard-Sibuya asymptotic expansion for $f$ and its derivatives, and, on the other hand, to the boundedness of the derivatives of $f$ on bounded proper subpolysectors of $S$.
\end{abstract}

2000 Mathematics subject classification: primary 34E05, 41 A60, $41 \mathrm{~A}$.

\section{Introduction}

Poincaré $[\mathrm{P}]$ put forward the notion of asymptotic expansion of a function $f$, defined and holomorphic on an open sector $S$ in the complex plane, as the variable tends to the vertex (which, without loss of generality, we will take to be the origin). A basic property is easily deduced from the definition:

(A) If a function $f$ admits asymptotic expansion, so they do its derivatives.

From this fact the following equivalence is obtained:

(B) $f$ admits asymptotic expansion if and only if its derivatives (including $f=f^{(0)}$ ) are bounded on bounded proper subsectors of $S$.

These results may be found in [W, pages 32-40].

When generalizing this concept for functions of several complex variables holomorphic on polysectors $S$ in $\mathbb{C}^{n}$, it seems reasonable to ask about the validity of (A)

(C) 2001 Australian Mathematical Society 0263-6115/2001 \$A2.00+0.00 
and (B). The different approaches in the literature come out of the diverse interpretations of what a partial sum of a series is when translated into several variables. Among those, the ones given by Gérard and Sibuya [GS] and Majima [M1] are outstanding. The aim of this paper is to give an example (the first in the literature, to our knowledge) showing that Gérard-Sibuya's definition satisfies neither property (A) nor (B), and to provide a complete proof of the equivalence of the following statements:

(i) the derivatives of $f: S \subset \mathbb{C}^{n} \rightarrow \mathbb{C}$ admit Gérard-Sibuya asymptotic expansion at $\mathbf{0}$;

(ii) the derivatives of $f$ are bounded on bounded proper subpolysectors of $S$;

(iii) $f$ is strongly asymptotically developable, that is, in the sense of Majima.

It is then clear that Majima's definition is more restrictive than Gérard-Sibuya's one, and it implies properties (A) and (B).

After some notation (Section 2), we devote Section 3 to the aforementioned example. Section 4 starts with some results relating the derivatives of a function $f$ to the coefficients of its Gérard-Sibuya asymptotic expansion (for brevity and from now on, GS-ae). Proposition 4.2 states that when $f$ and a certain derivative $D^{\beta} f$ both admit GS-ae, the second expansion is the formal partial derivative of order $\boldsymbol{\beta}$ of the first one; this improves a result in [GS, page 153], that used some additional hypotheses. We arrive so at the equivalence of (i) and (ii) (Theorem 4.4).

It is then natural to consider the space $\mathscr{A}(S)$ of complex functions holomorphic in a polysector $S \subset \mathbb{C}^{n}$ whose derivatives remain bounded on bounded proper subpolysectors of $S$. Actually, we will do all of our work on the space $\mathscr{A}(S, E)$ of functions defined and holomorphic on $S$, with values in a Fréchet space $E$, and satisfying the same boundedness condition. On the one hand, the results and their proofs in this setting do not essentially differ from the ones for $\mathscr{A}(S)$. On the other hand, since $\mathscr{A}(S)$ is a Fréchet space when given its natural topology, it makes sense to compare $\mathscr{A}(S \times U, \mathbb{C})$ and $\mathscr{A}(S, \mathscr{A}(U, \mathbb{C}))$, where $S$ and $U$ are polysectors in $\mathbb{C}^{n}$ and $\mathbb{C}^{m}$, respectively. These spaces are indeed isomorphic Fréchet spaces (Theorem 4.5), and one can determine the way differentiation interacts with the GS-ae of both a function $f \in \mathscr{A}(S \times U, E)$ and the corresponding $f^{*} \in \mathscr{A}(S, \mathscr{A}(U, E)$ ) (defined by the isomorphism).

In Section 5 we establish the link between the two notions of asymptotic expansion. Majima [M2] gives the concept of strongly asymptotic expansion for a function in terms of a so-called total family of related functions that satisfies certain 'consistency' properties. Haraoka [Ha] treated this kind of expansion with Gevrey-type bounds, and indicated the formulae (4), that show the elements of the total family as suitable limits of the derivatives of the function. This resembles similar expressions for the coefficients of the asymptotic expansion of a function in both the one variable case and the Gérard-Sibuya setting (Proposition 4.1). It turns out that these limits make 
perfect sense when applied to a function in $\mathscr{A}(S, E)$, and the functions they define form a family for which consistency easily appears; the equivalence of (ii) and (iii) follows (Theorem 5.2).

This work, as far as we know, is the first to establish in one place that both (i) and (iii) are equivalent to (ii). The techniques used are elementary, and have been useful in several instances. Firstly, the isomorphism in Theorem 4.5 allows us to constructively solve the corresponding Borel-Ritt-type problem in $\mathscr{A}(S, E)$, by applying statements valid for vector-valued functions on sectors, and induction on the number of variables [He, HS]. Also, the second author and Galindo [SG] have applied the equivalence of (ii) and (iii) (a proof of which is sketched in their paper) to solve this and a new BorelRitt type interpolation problem for functions on polysectors by a non-constructive functional-analytic procedure. Subsequently, Zurro [Z] proved that (i) and (iii) are equivalent with completely different techniques based on Whitney's theorem on $\mathscr{C}^{x}$ extensions.

\section{Notation}

Let $\mathbb{N}=\{0,1,2, \ldots\}$. For $n \in \mathbb{N}, n \geq 1$, put $N=\{1,2, \ldots, n\}$. Let $\alpha=\left(\alpha_{1}\right.$, $\left.\alpha_{2}, \ldots, \alpha_{n}\right), \beta=\left(\beta_{1}, \beta_{2}, \ldots, \beta_{n}\right) \in \mathbb{N}^{n}$ be two multiindices, $z=\left(z_{1}, z_{2}, \ldots, z_{n}\right)$ $\in \mathbb{C}^{\prime \prime}$, and $m$ a natural number. We set

$$
\begin{array}{ll}
m \boldsymbol{\alpha}=\left(m \alpha_{1}, m \alpha_{2}, \ldots, m \alpha_{n}\right), & \boldsymbol{\alpha}+\boldsymbol{\beta}=\left(\alpha_{1}+\beta_{1}, \alpha_{2}+\beta_{2}, \ldots, \alpha_{n}+\beta_{n}\right), \\
|\boldsymbol{\alpha}|=\alpha_{1}+\alpha_{2}+\cdots+\alpha_{n} . & \alpha !=\alpha_{1} ! \alpha_{2} ! \cdots \alpha_{n} !, \\
\alpha \leq \beta \Leftrightarrow \alpha_{j} \leq \beta_{j}, j \in N, & \boldsymbol{\alpha}<\boldsymbol{\beta} \Leftrightarrow \alpha_{j}<\beta_{j}, j \in N, \\
1=(1.1, \ldots, 1), & \boldsymbol{e}_{j}=(0 \ldots, 1 \ldots, 0), \\
z^{\alpha}=z_{1}^{\alpha_{1}} z_{2}^{\alpha_{2}} \ldots z_{n}^{\alpha_{n}}, & \left|z^{\alpha}\right|=|z|^{\alpha}=\left|z_{1}\right|^{\alpha_{1}}\left|z_{2}\right|^{\alpha_{2}} \ldots\left|z_{n}\right|^{\alpha_{n},}, \\
\|z\|=\left|z_{1}\right|+\left|z_{2}\right|+\cdots+\left|z_{n 1}\right|, & D^{\alpha}=\frac{\partial^{\alpha}}{\partial z^{\alpha}}=\frac{\partial^{|\alpha|}}{\partial z_{1}^{\alpha_{1}} \partial z_{2}^{\alpha_{2}} \cdots \partial z_{n}^{\alpha_{n}} .}
\end{array}
$$

If $J$ is a nonempty subset of $N$, the number of elements of $J$ will be \#J.

For $j=1,2 \ldots n$, consider an open sector in $\mathbb{C}$ with vertex at the origin,

$$
S_{j}=\left\{z \in \mathbb{C}: \theta_{1 j}<\arg (z)<\theta_{2 j}, 0<|z|<R_{j}\right\} .
$$

where $0<\theta_{2 j}-\theta_{1 j} \leq 2 \pi$ and $R_{j} \in(0,+\infty)$. The cartesian product of open sectors $S=\prod_{j=1}^{n} S_{j} \subset \mathbb{C}^{n}$ will be called (open) polysector in $\mathbb{C}^{n}$ with vertex at $\mathbf{0}$.

We say that a polysector $T=\prod_{j=1}^{n} T_{j}$ in $\mathbb{C}^{n}$ (with vertex at the origin) is a bounded proper subpolysector of $S$ if it is bounded and $\bar{T}, \subset S_{j} \cup\{0\}, j=1,2, \ldots, n$. In this case, we write $T \ll S$. 
If $J=\left\{j_{1}<j_{2}<\ldots<j_{k}\right\}$ is a nonempty subset of $N$ and $z=\left(z_{1}, \ldots, z_{n}\right) \in \mathbb{C}^{n}$, we put $z_{J}=\left(z_{j_{1}}, z_{j_{2}}, \ldots, z_{j_{k}}\right)$. Let $J$ and $L$ be nonempty disjoint subsets of $N$. For $z_{J} \in \mathbb{C}^{J}$ and $z_{L} \in \mathbb{C}^{L},\left(z_{J}, z_{L}\right)$ represents the element of $\mathbb{C}^{J \cup L}$ satisfying $\left(z_{J}, z_{L}\right)_{J}=$ $z_{J},\left(z_{J}, z_{L}\right)_{L}=z_{L}$; we also write $J^{c}=N-J$, and for $j \in N$ we use $j^{c}$ instead of $\{j\}^{c}$. In particular, we shall use these conventions for multiindices.

If $S=\prod_{j=1}^{n} S_{j}$ is a polysector of $\mathbb{C}^{n}$, then $S_{J}=\prod_{j \in J} S_{j} \subset \mathbb{C}^{J}$.

For $\delta \in(0, \infty)^{n}$, we denote by $D_{\delta}(z)$ (respectively, $\bar{D}_{\delta}(z)$ ) the open (respectively closed) polydisc centered at $z$ with polyradius $\delta ; \partial_{0} D_{\delta}(z)$ will be their distinguished boundary. In case $\delta=(\delta, \delta, \ldots, \delta)$, we simply write $D_{\delta}(z)$.

\section{Counterexample}

We begin with the notion of asymptotic expansion by Gérard-Sibuya [GS].

Let $S \subset \mathbb{C}^{n}$ be a polysector with vertex at 0 . A holomorphic function $f: S \rightarrow \mathbb{C}$ admits Gérard-Sibuya asymptotic expansion (briefly, GS-ae) at 0 if there exists a (formal) power series $\sum_{\alpha \in \mathbb{N}^{n}} a_{\alpha} z^{\alpha}$, with $a_{\alpha} \in \mathbb{C}$, such that for every $T \ll S$ and $m \in \mathbb{N}$ there exists a constant $C>0$ (depending on $T$ and $m$ ) such that

$$
\left|f(z)-\sum_{j=0}^{m} \sum_{|\alpha|=j} a_{\alpha} z^{\alpha}\right| \leq C\|z\|^{n+1}, \quad z \in T .
$$

If $f$ admits GS-ae, the series is unique, and it is said to asymptotically represent the function $f$, or to be the GS-ae of $f$. The basic properties obtainable from this definition can be found in [GS, pages 142-153].

We now give an example of a function that admits GS-ae, while none of its derivatives does. We begin with an easy lemma.

LEMMA 3.1. Let $S_{1}$ be a sector in $\mathbb{C}, S_{2}$ a polysector in $\mathbb{C}^{n-1}(n \geq 2)$. If $f_{1}: S_{1} \rightarrow \mathbb{C}$ is holomorphic in $S_{1}$ and bounded on every $T_{1} \ll S_{1}$, and $f_{2}: S_{2} \rightarrow \mathbb{C}$ is holomorphic in $S_{2}$ and admits the zero series as GS-ae, then $f: S_{1} \times S_{2} \rightarrow \mathbb{C}$ defined by $f\left(z_{1}, z_{2}\right)=$ $f_{1}\left(z_{1}\right) f_{2}\left(z_{2}\right),\left(z_{1}, z_{2}\right) \in S_{1} \times S_{2}$ admits the zero series as GS-ae.

In order to construct our function, we consider the sectors

$$
S_{1}=S_{2}=\cdots=S_{n}=\{z \in \mathbb{C}:|\arg (z)|<\pi,|z|>0\} \subset \mathbb{C},
$$

and the polysector $S=\prod_{j=1}^{n} S_{j} \subset \mathbb{C}^{n}$. By considering Blaschke products with suitably chosen zeroes (see [R, Chapter 15 , Problem 14, page 360]), one can show the existence of bounded holomorphic functions in $D_{1}(0)$, say $h_{0}: D_{1}(0) \rightarrow \mathbb{C}$, 
such that the radial limit, $\lim _{r \rightarrow 1, r<1} h_{0}(r)$, does not exist. A theorem of Lindelöf $[\mathrm{R}$, Theorem 12.10, page 294] implies that for any curve $\Gamma:[0,1] \rightarrow \bar{D}_{1}(0)$ such that

$$
|\Gamma(t)|<1 \quad \text { for } t \in[0,1) \quad \text { and } \quad \Gamma(1)=1 \text {, }
$$

the limit, $\lim _{t \rightarrow 1^{-}} h_{0}(\Gamma(t))$, does not exist either. Now the use of suitable conformal transformations allows us to deduce the existence of a function $h$ holomorphic and bounded in $S_{1}$ and such that no limit of the form

$$
\lim _{r \rightarrow 0, r>0} h\left(r \mathrm{e}^{i \theta}\right) \quad(|\theta|<\pi)
$$

exists. As a consequence, these 'directional' limits at 0 do not exist for the derivatives of $h$. Also, it is well known that we may find a function $g$ holomorphic in $S_{1}$, not identically zero and admitting the zero series as asymptotic expansion at 0 , the same being valid for the derivatives of $g$ (for example, $g(z)=\exp \left(-1 / z^{1 / 8}\right)$, where the principal value of the root is considered). So, for every $H \ll S_{1}$ and $m \in \mathbb{N}$ we have

$$
\sup \left\{\left|g^{(m)}(t)\right|: t \in H\right\}<\infty .
$$

We recall that $S_{j c}=S_{1} \times \cdots \times S_{j-1} \times S_{j+1} \times \cdots \times S_{n}$. For $j=1,2, \ldots, n$, define $G_{j}: S_{j} \rightarrow \mathbb{C}$ as

$$
G_{j}\left(z_{j}\right)=G_{j}\left(z_{1}, \ldots, z_{j-1}, z_{j+1}, \ldots, z_{n}\right)=\prod_{\substack{i=1 \\ i \neq j}}^{n} g\left(z_{i}\right) .
$$

$G_{j}$ is holomorphic and, according to the previous lemma, admits the zero series as GS-ae. For the same reason, the function

$$
f\left(z_{1}, z_{2}, \ldots, z_{n}\right)=\sum_{j=1}^{n} h\left(z_{j}\right) G_{j}\left(z_{j}\right),
$$

defined and holomorphic in $S$, has again the zero series as GS-ae. $f$ is the desired function. Suppose that for some $\boldsymbol{\alpha}_{0}=\left(\alpha_{1}, \alpha_{2}, \ldots, \alpha_{n}\right) \in \mathbb{N}^{n} \backslash\{\mathbf{0}\}, D^{\boldsymbol{\alpha}_{0}} f$ admits GS-ae. Then, given $T=\prod_{j=1}^{n} T_{j} \ll S$ we have

$$
\sup \left\{\left|D^{\boldsymbol{\alpha}_{0}} f(z)\right|: z \in T\right\}<\infty \text {. }
$$

There exists $k \in\{1,2, \ldots, n\}$ such that $\alpha_{k} \geq 1$, and for $j \neq k$ we can choose $t_{j} \in T_{j}$ with $g^{\left(\alpha_{,}\right)}\left(t_{j}\right) \neq 0$. Then, for every $t \in T_{k}$ we have

$$
D^{\alpha_{1}} f\left(t_{1}, \ldots, t, \ldots, t_{n}\right)=h^{\left(\alpha_{k}\right)}(t) \prod_{\substack{i=1 \\ i \neq k}}^{n} g^{\left(\alpha_{i}\right)}\left(t_{i}\right)+\sum_{\substack{j=1 \\ j \neq k}}^{n} h^{\left(\alpha_{j}\right)}\left(t_{j}\right) g^{\left(\alpha_{k}\right)}(t) \prod_{\substack{i=1 \\ i \neq j . k}} g^{\left(\alpha_{i}\right)}\left(t_{i}\right) .
$$

From (1) and (2) we deduce that $\sup \left\{\left|h^{\left(\alpha_{k}\right)}(t)\right|: t \in T_{k}\right\}<\infty$, which implies that $h^{\left(\alpha_{t}-1\right)}$ has a finite limit at 0 along any direction on $T_{k}$. The way $h$ was chosen makes this a contradiction. 


\section{The space $\mathscr{A}(S, E)$}

Let $S \subset \mathbb{C}^{n}$ be a polysector with vertex at $\mathbf{0}, E$ a Fréchet space, and $f: S \rightarrow E$ holomorphic. We call $\hat{E}$ the set of continuous seminorms defined on $E$. We say $f$ admits GS-ae at 0 if there exists a (formal) power series $\sum_{\alpha \in \mathbb{N}^{n}} a_{\alpha} z^{\alpha}$, with $a_{\alpha} \in E$, such that for every $p \in \hat{E}, T \ll S$ and $m \in \mathbb{N}$, there exists a constant $C>0$ (depending on $p, T$ and $m$ ) such that

$$
p\left(f(z)-\sum_{j=0}^{m} \sum_{|\alpha|=j} a_{\alpha} z^{\alpha}\right) \leq C\|z\|^{m+1}, \quad z \in T .
$$

In this situation, we write

$$
f \sim_{S} \sum_{\alpha \in \mathbb{N}^{n}} a_{\alpha} z^{\alpha}, \quad z \rightarrow \mathbf{0},
$$

or simply $f \sim \sum_{\alpha \in \mathbb{N}^{n}} a_{\alpha} z^{\alpha}$ when there is no ambiguity.

PROPOSITION 4.1. If $\sim \sum_{\alpha \in \mathbb{N}^{n}} a_{\alpha} z^{\alpha}$, then for every compact $K \subset S$ and $\alpha \in \mathbb{N}^{n}$,

$$
\lim _{z \rightarrow 0 . z \in \widetilde{K}} \frac{D^{\alpha} f(z)}{\alpha !}=a_{\alpha},
$$

where $\widetilde{K}=\{\lambda z: \lambda \in(0,1], z \in K\}$.

PROOF. Put $|\boldsymbol{\alpha}|=m$ and $g(z)=\sum_{j=0}^{m} \sum_{|\alpha|=j} a_{\alpha} z^{\alpha}, z \in S$. Consider $T \ll S$ such that $K \subset T$. If $d=\operatorname{dist}\left(K, \mathbb{C}^{n}-T\right)$ and $M=\max \{\|z\|: z \in K\}$, take $r=d /(2 M n)$; it is easy to check that for every $z \in K$ we have $\bar{D}_{r\|z\|}(z) \subset T$.

Given $p \in \hat{E}$ and $\varepsilon>0$, there exists $\rho>0$ such that for $\omega \in \bar{D}_{\rho}(\mathbf{0}) \cap T$ we have

$$
p\left(\frac{f(\omega)-g(\omega)}{\|\omega\|^{m}}\right)<\varepsilon .
$$

If we choose $\delta=\rho /(n(1+n r))$, then $\bar{D}_{r\|z\|}(z) \subset \bar{D}_{\rho}(\mathbf{0}) \cap T$ whenever $z \in D_{\delta}(\mathbf{0}) \cap \widetilde{K}$. So, for such $z$ we get

$$
\frac{D^{\alpha} f(z)}{\alpha !}-a_{\alpha}=\frac{D^{\alpha} f(z)-D^{\alpha} g(z)}{\alpha !}=\frac{1}{(2 \pi i)^{n}} \int_{i_{k_{0}} D_{r ; i \mid 1 !}(z)} \frac{f(\omega)-g(\omega)}{(\omega-z)^{\alpha+1}} d \omega,
$$

and hence

$$
\begin{aligned}
& p\left(\frac{D^{\alpha} f(z)}{\alpha !}-a_{\alpha}\right) \leq \frac{(2 \pi r\|z\|)^{n}}{(2 \pi)^{n}(r\|z\|)^{m+n}} \sup _{\omega \in i_{0} D_{r|||\||}(z)} p(f(\omega)-g(\omega)) \\
& \leq \frac{1}{(r\|z\|)^{m}} \sup _{\boldsymbol{\omega} \in i_{n} D_{n \|: 1}(z)} p\left(\frac{f(\boldsymbol{\omega})-g(\boldsymbol{\omega})}{\|\boldsymbol{\omega}\|^{m}}\right)\|\boldsymbol{\omega}\|^{m}<\frac{(1+n r)^{m}}{r^{m}} \varepsilon,
\end{aligned}
$$

which concludes the proof. 
We now improve a result of Gérard-Sibuya [GS, Corollary 2.2.2, page 153]. As made clear by the example in Section 3, the fact that $f$ admits GS-ae says nothing about $D^{\beta} f$. However, if $D^{\beta} f$ also admits GS-ae, we can be sure that it is the one we expect.

PROPOSITION 4.2. Suppose that $f \sim \sum_{\alpha \in \mathbb{N}^{n}} a_{\alpha} z^{\alpha}$, and that for some $\beta \in \mathbb{N}^{n}$, $D^{\beta} f \sim \sum_{\alpha \in \mathbb{N}^{n}} b_{\alpha} z^{\alpha}$. Then, $b_{\alpha}=(\boldsymbol{\alpha}+\boldsymbol{\beta}) ! a_{\alpha+\beta} / \boldsymbol{\alpha} !, \boldsymbol{\alpha} \in \mathbb{N}^{n}$.

PROOF. Consider a compact subset $K$ of $S$. By the previous proposition, for every $\alpha \in \mathbb{N}^{n}$ we have

$$
b_{\alpha}=\lim _{\substack{z \rightarrow 0 \\ z \in \widetilde{K}}} \frac{D^{\alpha}\left(D^{\beta} f\right)(z)}{\alpha !}=\frac{(\alpha+\beta) !}{\alpha !} \lim _{\substack{z \rightarrow 0 \\ z \in \widetilde{K}}} \frac{D^{\alpha+\beta} f(z)}{(\alpha+\beta) !}=\frac{(\alpha+\beta) !}{\alpha !} a_{\alpha+\beta} .
$$

We now turn to the problem of finding out what conditions should be imposed on $f$ to ensure that $D^{\beta} f$ admits GS-ae for every $\boldsymbol{\beta} \in \mathbb{N}^{n}$. If this were the case, we observe, as an easy consequence of the very definition, that $D^{\beta} f$ would be bounded on every $T \ll S$. This is indeed sufficient.

PROPOSITION 4.3. Let $f: S \rightarrow E$ be holomorphic and such that for every $p \in \hat{E}$, $\alpha \in \mathbb{N}^{n}$ and $T \ll S$, we have $\sup \left\{p\left(D^{\alpha} f(z)\right): z \in T\right\}<\infty$. Then, for every $\alpha \in \mathbb{N}^{n}$ and $T \ll S$ there exists the limit

$$
\lim _{\substack{z \rightarrow 0 \\ z \in T}} D^{\alpha} f(z)=\alpha ! a_{\alpha} \in E,
$$

and for every $\boldsymbol{\beta} \in \mathbb{N}^{n}, D^{\beta} f \sim \sum_{\boldsymbol{\alpha} \in \mathbb{N}^{n}}((\boldsymbol{\alpha}+\boldsymbol{\beta}) ! / \boldsymbol{\alpha} !) a_{\boldsymbol{\alpha}+\boldsymbol{\beta}} z^{\alpha}$.

PROOF. For the first part, and without loss of generality, we will argue only with $T=$ $\prod_{j=1}^{n} T_{j} \ll S$, with $T_{j}$ of aperture less than $\pi$. It is enough to prove that $\left\{D^{\alpha} f\left(z_{m}\right)\right\}_{m=1}^{\infty}$ is a Cauchy sequence in $E$ for any sequence $\left\{z_{m}\right\}_{m=1}^{\infty} \subset T$ with $\lim _{m \rightarrow \infty} z_{m}=\mathbf{0}$. Put $z_{m}=\left(z_{m 1}, z_{m 2}, \ldots, z_{m n}\right), m \geq 1$. Barrow's formula allows us to write

$$
D^{\alpha} f\left(z_{r}\right)-D^{\alpha} f\left(z_{s}\right)=\sum_{j=1}^{n} \int_{0}^{1} D^{\alpha+e_{j}} f\left((1-t) z_{s}+t z_{r}\right)\left(z_{r j}-z_{s j}\right) d t .
$$

Let $p \in \hat{E}$. If we take $M=\max _{1 \leq j \leq n} \sup _{z \in T} p\left(D^{\alpha+e_{j}} f(z)\right)<\infty$, we have

$$
p\left(D^{\alpha} f\left(z_{r}\right)-D^{\alpha} f\left(z_{s}\right)\right) \leq M\left\|z_{r}-z_{s}\right\|,
$$

and the conclusion easily follows.

For the second part, let us note that for $\boldsymbol{\beta} \in \mathbb{N}^{n}-\{\mathbf{0}\}$ the derivatives of $D^{\beta} f$ are among those of $f$, so that the former are all bounded on every $T \ll S$. So, if we prove 
that $f$ admits GS-ae (and that this is $\sum_{\alpha \in \mathbb{N}^{n}} a_{\alpha} z^{\alpha}$ ), the same argument would apply in order to prove that $D^{\beta} f$ also admits GS-ae. The previous proposition allows us to conclude the proof.

Consider $m \in \mathbb{N}, T \ll S$ and a decreasing sequence $\left\{\lambda_{j}\right\}_{j=1}^{\infty} \subset[0,1)$ with $\lim _{j \rightarrow \infty} \lambda_{j}=0$. For $z \in T$ and $j=1,2, \ldots$, Taylor's formula gives

$$
\begin{aligned}
f(z)= & \sum_{k=0}^{m} \sum_{|\alpha|=k} \frac{D^{\alpha} f\left(\lambda_{j} z\right)}{\alpha !}\left(z-\lambda_{j} z\right)^{\alpha} \\
& +(m+1) \int_{0}^{1}(1-t)^{m} \sum_{|\alpha|=m+1} \frac{D^{\alpha} f\left((1-t) \lambda_{j} z+t z\right)}{\alpha !}\left(z-\lambda_{j} z\right)^{\alpha} d t .
\end{aligned}
$$

Letting $j \rightarrow \infty$, we find

$$
f(z)=\sum_{k=0}^{m} \sum_{|\alpha|=k} a_{\alpha} z^{\alpha}+(m+1) \int_{0}^{1}(1-t)^{m} \sum_{|\alpha|=m+1} \frac{D^{\alpha} f(t z)}{\alpha !} z^{\alpha} d t .
$$

Since $\sum_{|\alpha|=m+1}\left|z^{\alpha}\right| \leq\|z\|^{m+1}$, for $p \in \hat{E}$ we have

$$
p\left(f(z)-\sum_{k=0}^{m} \sum_{|\boldsymbol{\alpha}|=k} a_{\alpha} z^{\alpha}\right) \leq(m+1) M\|z\|^{m+1}, \quad z \in T,
$$

where $M=\max _{|\alpha|=m+1} \sup _{z \in T} p\left(D^{\alpha} f(z) / \alpha !\right)$.

In view of this result, we introduce the complex vector space $\mathscr{A}(S, E)$ consisting of the holomorphic functions $f: S \rightarrow E$ such that for every $p \in \hat{E}, \boldsymbol{\alpha} \in \mathbb{N}^{n}$ and $T \ll S$ we have

$$
Q_{p, \boldsymbol{\alpha}, T}(f)=\sup \left\{p\left(D^{\alpha} f(z)\right): z \in T\right\}<\infty .
$$

We have already proved

THEOREM 4.4. $f \in \mathscr{A}(S, E)$ if and only if $D^{\alpha} f$ admits GS-ae for every $\alpha$.

$\mathscr{A}(S, E)$ is a differential algebra, which equipped with the topology generated by the family $\left\{Q_{p, \alpha, T}\right\}$ of seminorms is a Fréchet space.

The proof of the following result is postponed until the last section, in order not to interrupt the course of the ideas.

THEOREM 4.5. (i) The map

$$
f \in \mathscr{A}(S \times U, E) \rightarrow f^{*} \in \mathscr{A}(S, \mathscr{A}(U, E)),
$$


defined for every $z \in S$ by $f^{*}(z)=f(z, \cdot)$, is a topological isomorphism, and for every $\alpha \in \mathbb{N}^{n}$ and $\beta \in \mathbb{N}^{m}$ we have

$$
D^{(\alpha, \beta)} f(z, \omega)=D^{\beta}\left(D^{\alpha} f^{*}(z)\right)(\omega), \quad(z, \omega) \in S \times U .
$$

(ii) Let $f \in \mathscr{A}(S \times U, E)$. For $\omega \in U, \alpha \in \mathbb{N}^{n}$ and $T \ll S$,

$$
\lim _{\substack{z \rightarrow 0 \\ z \in T}} \frac{D^{(\alpha, 0)} f(z, \omega)}{\alpha !}=f_{(\boldsymbol{\alpha}, 0)}(\boldsymbol{\omega})
$$

exists, the convergence being uniform on every $V \ll U$, and defines a function $f_{(\alpha, 0)} \in \mathscr{A}(U, E)$.

(iii) For every $\boldsymbol{\gamma} \in \mathbb{N}^{m},\left(D^{(\mathbf{0}, \gamma)} f\right)^{*} \sim_{s} \sum_{\boldsymbol{\alpha} \in \mathbb{N}^{n}}\left(D^{\gamma} f_{(\boldsymbol{\alpha}, 0)}\right) z^{\alpha}$.

\section{Strongly asymptotically developable functions}

Although the results in this section are sketched in [SG], we supply here their detailed proofs, based on the previous material.

Let $f \in \mathscr{A}(S, E)$. According to (ii) in Theorem 4.5, if $\emptyset \neq J \subset N$ and $\boldsymbol{\alpha}_{J} \in \mathbb{N}^{J}$, we can define a function from $S_{J}$, to $E$ by

$$
f_{\alpha_{j}}\left(z_{J^{c}}\right)=\lim _{\substack{z_{J} \rightarrow 0 \\ z j \in T_{j}}} \frac{D^{\left(\alpha_{J}, \boldsymbol{o}^{\prime c}\right)} f(z)}{\alpha_{J} !}, \quad z_{J^{c}} \in S_{J^{c}},
$$

for any subpolysector $T_{J}$ of $S_{J} ; f_{\alpha} \in \mathscr{A}\left(S_{\jmath c}, E\right)$ (setting $\mathscr{A}\left(S_{N^{c}}, E\right)=E$ ).

In this way, we may associate with $f$ a family

$$
\mathscr{F}(f)=\left\{f_{\alpha}, \mathscr{A}\left(S_{J^{\prime}}, E\right): \emptyset \neq J \subset N, \alpha_{J} \in \mathbb{N}^{J}\right\} .
$$

PROPOSITION 5.1 (Consistency conditions). Let $f \in \mathscr{A}(S, E)$. Then, for every pair of disjoint nonempty subsets $J$ and $L$ of $N$, for $\boldsymbol{\alpha}_{J} \in \mathbb{N}^{J}$ and $\boldsymbol{\alpha}_{L} \in \mathbb{N}^{L}$, and for $T_{L} \ll S_{L}$,

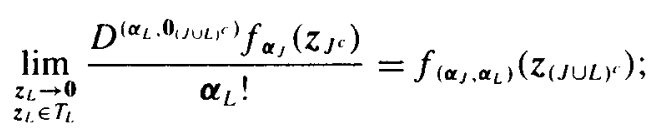

the limit is uniform on bounded proper subpolysectors of $S_{(J \cup L)}$ whenever $J \cup L \neq N$.

Proof. We only deal with the case $J \cup L \neq N$. By the definition of $\mathscr{F}(f)$, for $T_{J} \ll S_{J}$ and $T_{L} \ll S_{L}$ we have

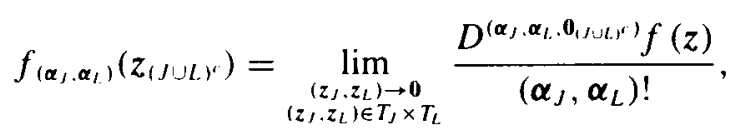


uniformly on every $T_{(J \cup L)^{c}} \ll S_{(J \cup L)^{c}}$. The function $\left(D^{\left(\alpha_{L}, \mathbf{0}_{L} c\right)} f\right)_{J}^{*}$, defined from $S_{J}$ to $\mathscr{A}\left(S_{J c}, E\right)$ by

$$
\left(D^{\left(\alpha_{L}, 0_{L c}\right)} f\right)_{J}^{*}\left(z_{J}\right)\left(z_{J c}\right)=D^{\left(\alpha_{L}, 0_{\left.L^{c}\right)}\right.} f\left(z_{J}, z_{J c}\right),
$$

admits the series $\sum_{\alpha_{J} \in \mathbb{N}^{\prime}} D^{\left(\alpha_{L}, 0_{s c}\right)} f_{\alpha_{\jmath}} z_{J}^{\alpha_{J}}$ as GS-ae (see Theorem 4.5 (iii)); so, Proposition 4.3 implies that

$$
\lim _{\substack{z, \rightarrow 0 \\ z \in T_{J}}} \frac{D^{\alpha_{J}}\left(\left(D^{\left(\boldsymbol{\alpha}_{L}, \mathbf{0}_{L^{c}}\right)} f\right)_{J}^{*}\right)\left(z_{J}\right)}{\boldsymbol{\alpha}_{J} !}=D^{\left(\boldsymbol{\alpha}_{L}, \mathbf{0}_{I J U L^{\prime}}\right)} f_{\alpha_{J}} \quad \text { in } \mathscr{A}\left(S_{J^{c}}, E\right) .
$$

By (3) in Theorem 4.5, this translates into

$$
\lim _{\substack{z, \rightarrow 0 \\ z J \in T_{J}}} \frac{D^{\left(\boldsymbol{\alpha}_{J}, \boldsymbol{\alpha}_{L}, \mathbf{0}_{J} J U L^{c}\right)} f(z)}{\boldsymbol{\alpha}_{J} !}=D^{\left(\alpha_{L}, \mathbf{0}_{(J U L,}\right)} f_{\alpha_{J}}\left(z_{J c}\right),
$$

uniformly on every $T_{J c} \ll S_{J c}$. From (6) and (7) the conclusion follows.

Hereafter, we will say that a family

$$
\mathscr{F}=\left\{f_{\alpha^{\prime}} \in \mathscr{A}\left(S_{J^{r}}, E\right): \emptyset \neq J \subset N, \boldsymbol{\alpha}_{J} \in \mathbb{N}^{J}\right\},
$$

or briefly $\mathscr{F}=\left\{f_{\alpha_{j}}\right\}$, is consistent if it satisfies (5).

We adapt the concept of strongly asymptotic expansion (see [M1]) to our context.

Let $f: S \rightarrow E$ be holomorphic. We say that $f$ is strongly asymptotically developable at $\mathbf{0}$ if there exists a family

$$
\mathscr{F}=\left\{f_{\boldsymbol{\alpha}}: \emptyset \neq J \subset N, \boldsymbol{\alpha}_{J} \in \mathbb{N}^{J}\right\},
$$

where $f_{\alpha}$ is a holomorphic function from $S_{j}$ to $E$ when $J \neq N$, and $f_{\alpha} \in E$ when $J=N$, such that the following holds: if we define

$$
\operatorname{App}_{\boldsymbol{\alpha}}(\mathscr{F})(z)=\sum_{\boldsymbol{\beta} \neq J \subset N}(-1)^{\# J+1} \sum_{\substack{\beta_{j} \in \mathbb{N}^{J} \\ \beta_{j} \leq \alpha_{j}-1}} f_{\beta_{J}}\left(z_{J c}\right) z_{J}^{\beta_{J}}, \quad \alpha \in \mathbb{N}^{n}, z \in S,
$$

then for every $p \in \hat{E}, T \ll S$ and $\alpha \in \mathbb{N}^{n}$,

$$
\sup \left\{p\left(\frac{f(z)-\operatorname{App}_{\alpha}(\mathscr{F})(z)}{z^{\alpha}}\right): z \in T\right\}<\infty .
$$

$\mathscr{F}$ will be called a total family of strongly asymptotic expansion associated with $f$, and will be denoted by $T A(f)$. The function $\operatorname{App}_{\alpha}(\mathscr{F}): S \rightarrow E$ is called the approximate function of order $\alpha$ corresponding to the family $\mathscr{F}$. 
THEOREM 5.2. Let $f: S \rightarrow E$ be holomorphic. Then $f$ is strongly asymptotically developable at 0 if and only if $\in \mathscr{A}(S, E)$. If this is the case, then $\mathscr{F}(f)=T A(f)$.

ProOf. Assume $f$ is strongly asymptotically developable. Fix $T \ll S$ and $\alpha \in \mathbb{N}^{n}$. We may take $T_{1} \ll S$ such that $T \ll T_{1}$, which allows us to find $r>0$ such that for every $z \in T$ the polydisc $\bar{D}_{r(z)}(z)$, with polyradius $\boldsymbol{r}(z)=\left(r\left|z_{1}\right|, r\left|z_{2}\right|, \ldots, r\left|z_{n}\right|\right) \epsilon$ $(0, \infty)^{n}$, is contained in $T_{1}$. If $\omega$ belongs to $\partial_{0} \bar{D}_{r(z)}(z)$, then $|\omega|^{\alpha} \leq(1+r)^{|\alpha|}|z|^{\alpha}$ and $|\omega-z|^{\alpha+1}=r^{|\alpha|+n}|z|^{\alpha+1}$. As $f$ admits a strongly asymptotic expansion at $\mathbf{0}$, given $p \in \hat{E}$ there exists $C=C_{p, T_{1}, \alpha}>0$ such that

$$
p\left(f(z)-\operatorname{App}_{\alpha}(T A(f))(z)\right) \leq C|z|^{\alpha}, \quad z \in T_{1} .
$$

Since $D^{\alpha} \operatorname{App}_{\alpha}(T A(f)) \equiv 0$ on $S$, we can apply Cauchy's integral formula to obtain that, for every $z \in T$,

$$
\begin{aligned}
p\left(D^{\alpha} f(z)\right) & =p\left(\frac{\alpha !}{(2 \pi i)^{n}} \int_{\partial_{0} \bar{D}_{r(z)}(z)} \frac{f(\omega)-\operatorname{App}_{\alpha}(T A(f))(\omega)}{(\omega-z)^{\alpha+1}} d \omega\right) \\
& \leq \alpha ! C\left(\frac{1+r}{r}\right)^{|\alpha|}<\infty
\end{aligned}
$$

and we deduce that $f \in \mathscr{A}(S, E)$.

Conversely, let $f \in \mathscr{A}(S, E)$. The error formula

$$
\begin{aligned}
(z)- & \operatorname{App}_{\alpha}(\mathscr{F}(f))(z) \\
& =\prod_{\substack{j=1 \\
\alpha_{j} \neq 0}}^{n}\left(\int_{0}^{z j} d t_{j .1} \int_{0}^{t_{j .1}} d t_{j .2} \cdots \int_{0}^{t_{j, \alpha_{j}-1}} d t_{j, \alpha_{j}}\right) D^{\alpha} f\left(t_{1, \alpha_{1}}, t_{2, \alpha_{2}}, \ldots, t_{n, \alpha_{n}}\right)
\end{aligned}
$$

was given by Haraoka [Ha]; a proof of it, adaptable to our setting, can be found in [Z]. For every $T \ll S, p \in \hat{E}$ and $\alpha \in \mathbb{N}^{n}$, $\sup _{z \in T} p\left(D^{\alpha} f(z)\right)=C_{p, T, \alpha}<\infty$. Then, for $z \in T$ we have

$$
p\left(f(z)-\operatorname{App}_{\alpha}(\mathscr{F}(f))(z)\right) \leq \frac{|z|^{\alpha}}{\alpha !} C_{p . T, \alpha},
$$

so $f$ is strongly asymptotically developable at $\mathbf{0}$, and $T A(f)=\mathscr{F}(f)$.

By (4), $T A(f)$ is unique. For $\emptyset \neq J \subset N$ and $\alpha_{J} \in \mathbb{N}^{J}, f_{\alpha_{J}} \in \mathscr{A}\left(S_{J^{\prime}}, E\right)$, thus the elements of $T A(f)$ are strongly asymptotically developable, and from the consistency conditions we see that

$$
T A\left(f_{\alpha_{s}}\right)=\left\{f_{\left(\boldsymbol{\alpha}_{j}, \beta_{L}\right)}: \emptyset \neq L \subset J^{c}, \boldsymbol{\beta}_{L} \in \mathbb{N}^{L}\right\} .
$$

We note that the notion of consistent family was given by Majima [M2, Part I, page 25], though we have arrived at it from a different setting. 


\section{Proof of Theorem 4.5}

(i) If $f \in \mathscr{A}(S \times U, E)$, then for each $z \in S$ the derivatives of $f(z, \cdot)$ (which is obviously holomorphic) are among those of $f$. So, $f(z, \cdot) \in \mathscr{A}(U, E)$.

We now prove that $f^{*}$ is partially holomorphic in $S$ (and, by Hartogs' theorem, holomorphic in $S$ ). Take $j \in\{1,2, \ldots, n\}$. Since $\partial f / \partial z_{j}$ belongs to $\mathscr{A}(S \times U, E)$, for every $s=\left(s_{1}, \ldots, s_{n}\right) \in S$ we have $\left(\partial f / \partial z_{j}\right)(s, \cdot) \in \mathscr{A}(U, E)$. This function is the $j$-th partial derivative of $f^{*}$ at $s$. Indeed, choose $T=\prod_{j=1}^{n} T_{j} \ll S$ with $s \in T$. There exists $\delta>0$ such that $\bar{B}_{\delta}\left(s_{j}\right) \subset S_{j}$; for any $p \in \hat{E}, \boldsymbol{\beta} \in \mathbb{N}^{m}, V \ll U$ and $u \in B_{\delta}(0), u \neq 0$, one has

$$
\begin{aligned}
Q_{p, \beta, V} & \left(\frac{f^{*}\left(s+u e_{j}\right)-f^{*}(s)}{u}-\left(\frac{\partial f}{\partial z_{j}}\right)(s, \cdot)\right) \\
& =\sup _{\omega \in V} p\left(D^{\beta}\left(\frac{f^{*}\left(s+u e_{j}\right)-f^{*}(s)}{u}\right)(\omega)-D^{\beta}\left(\left(\frac{\partial f}{\partial z_{j}}\right)(s, \cdot)\right)(\omega)\right) \\
& =\sup _{\omega \in V} p\left(\frac{1}{u}\left(D^{(0, \beta)} f\left(s+u e_{j}, \omega\right)-D^{(0, \beta)} f(s, \omega)\right)-D^{\left(\boldsymbol{e}_{j}, \beta\right)} f(s, \omega)\right) .
\end{aligned}
$$

We may write

$$
\begin{aligned}
\frac{1}{u}\left(D^{(0, \beta)} f\left(s+u e_{j}, \omega\right)-D^{(0, \beta)} f(s, \omega)\right)-D^{\left(e_{j}, \beta\right)} f(s, \omega) \\
\quad=\frac{1}{u} \int_{[0, u]}\left(D^{\left(e_{j}, \beta\right)} f\left(s+\xi e_{j}, \omega\right)-D^{\left(e_{j}, \beta\right)} f(s, \omega)\right) d \xi \\
\quad=\frac{1}{u} \int_{[0, u]}\left(\int_{[0, \xi]} D^{\left(2 e_{j}, \beta\right)} f\left(s+\eta \boldsymbol{e}_{j}, \omega\right) d \eta\right) d \xi, \quad \omega \in U
\end{aligned}
$$

and so

$$
\begin{aligned}
& Q_{p, \beta, V}\left(\frac{f^{*}\left(s+u e_{j}\right)-f^{*}(s)}{u}-\left(\frac{\partial f}{\partial z_{j}}\right)\right) \\
& \leq Q_{p,\left(2 e_{j}, \beta\right), T \times V}(f) \frac{1}{|u|} \int_{[0, u]}\left(\int_{[0, \xi]}|d \eta|\right)|d \xi|=\frac{1}{2} Q_{p,\left(2 e_{j}, \beta\right), T \times V}(f)|u|,
\end{aligned}
$$

from which the conclusion follows.

In order to obtain (3), consider for each $(z, \omega) \in S \times U$ a polydisc $D=D(z) \times D(\omega)$ centered at $(z, \omega)$ and contained in $S \times U$. Fubini's theorem and Cauchy's integral formula allow us to write

$$
D^{(\boldsymbol{\alpha}, \boldsymbol{\beta})} f(z, \boldsymbol{\omega})=\frac{\boldsymbol{\alpha} ! \boldsymbol{\beta} !}{(2 \pi i)^{n+m}} \int_{\partial_{0} D} \frac{f(\boldsymbol{\sigma}, \eta)}{(\boldsymbol{\alpha}-z)^{\alpha+1}(\eta-\omega)^{\beta+1}} d \sigma d \eta
$$




$$
\begin{aligned}
& =\frac{\beta !}{(2 \pi i)^{m}} \int_{\partial_{0} D(\omega)} \frac{1}{(\eta-\omega)^{\beta+1}} \cdot\left(\frac{\alpha !}{(2 \pi i)^{n}} \int_{i_{0} D(z)} \frac{f^{*}(\sigma)(\eta)}{(\sigma-z)^{\alpha+1}} d \sigma\right) d \eta \\
& =\frac{\beta !}{(2 \pi i)^{m}} \int_{\partial_{0} D(\omega)} \frac{1}{(\eta-\omega)^{\beta+1}}\left(D^{\alpha} f^{*}(z)(\eta)\right) d \eta=D^{\beta}\left(D^{\alpha} f^{*}(z)\right)(\omega) .
\end{aligned}
$$

From this equality we see that

$$
Q_{Q_{p, \beta, \gamma, \boldsymbol{\alpha}, T}}\left(f^{*}\right)=Q_{p,(\boldsymbol{\alpha}, \boldsymbol{\beta}), T \times V}(f)
$$

for every $p \in \hat{E},(\boldsymbol{\alpha}, \boldsymbol{\beta}) \in \mathbb{N}^{n+m}, T \ll S$ and $V \ll U$. So, $f^{*} \in \mathscr{A}(S, \mathscr{A}(U, E))$.

Conversely, given $f^{*} \in \mathscr{A}(S, \mathscr{A}(U, E))$, for every $z \in S$ the map $f^{*}(z)(\cdot)=$ $f(z, \cdot)$ is holomorphic in $U$. Also, for $s \in S$ there exists $\delta \in(0, \infty)^{n}$ such that whenever $z \in D_{\delta}(s) \subset S$ we have $f^{*}(z)=\sum_{\alpha \in \mathbb{N}^{n}} D^{\alpha} f^{*}(s)(z-s)^{\alpha} / \alpha$ !. Then, for every $\omega \in U$ we may write

$$
f(z, \omega)=f^{*}(z)(\boldsymbol{\omega})=\sum_{\alpha \in \mathbb{N}^{n}} \frac{D^{\alpha} f^{*}(s)(\omega)}{\alpha !}(z-s)^{\alpha},
$$

so that $f(\cdot, \boldsymbol{\omega})$ is holomorphic in $S$. Hence, $f$ is partially holomorphic. Reasoning as before, we obtain again (3) and (8), so that $f \in \mathscr{A}(S \times U, E)$.

Equality (8) implies that $f \rightarrow f^{*}$ is a topological isomorphism.

(ii) Throughout (ii) we fix $T \ll S, V \ll U, p \in \hat{E}$ and $\boldsymbol{\alpha} \in \mathbb{N}^{n}$. Arguing as in Proposition 4.3, we find $M>0$ (depending on $T, V, p$ and $\alpha$ ) such that if $\left\{z_{k}\right\}_{k=0}^{\infty} \subset T$ converges to $\mathbf{0}$, then

$$
p\left(D^{(\alpha .0)} f\left(z_{k}, \omega\right)-D^{(\alpha, 0)} f\left(z_{j}, \omega\right)\right) \leq M\left\|z_{k}-z_{j}\right\|, \quad k, j \in \mathbb{N}, \omega \in V,
$$

from which the existence and uniformity of the limits $f_{(\alpha, 0)}$ easily follows. Of course, these limits are independent of $T$. For $\left\{z_{k}\right\}_{k=0}^{\infty}$ as before, define $g_{k}(\boldsymbol{\omega})=D^{(\boldsymbol{\alpha}, 0)} f\left(z_{k}, \boldsymbol{\omega}\right)$, $k \in \mathbb{N}, \omega \in U$. Clearly, $g_{k} \in \mathscr{A}(U, E)$. Again, for $\boldsymbol{\beta} \in \mathbb{N}^{m}$ there exists a constant $M>0$ (depending on $\boldsymbol{\alpha}, \boldsymbol{\beta}, p, T$ and $V$ ) such that for every $\boldsymbol{\omega} \in V$ we have

$$
p\left(D^{(\boldsymbol{\alpha}, \boldsymbol{\beta})} f\left(z_{k}, \boldsymbol{\omega}\right)-D^{(\boldsymbol{\alpha}, \boldsymbol{\beta})} f\left(z_{j}, \boldsymbol{\omega}\right)\right) \leq M\left\|z_{k}-z_{j}\right\|, \quad k, j \in \mathbb{N},
$$

and so $Q_{p . \beta . V}\left(g_{k}-g_{j}\right) \leq M\left\|z_{k}-z_{j}\right\|$. Since $\mathscr{A}(U, E)$ is complete, $\left\{g_{k}\right\}_{k=0}^{\infty}$ converges to some $g \in \mathscr{A}(U, E)$, and

$$
g(\omega)=\lim _{k \rightarrow \infty} g_{k}(\omega)=\lim _{\substack{z \rightarrow 0 \\ z \in T}} D^{(\alpha, 0)} f(z, \omega)=\alpha ! f_{(\alpha, 0)}(\omega), \quad \omega \in U .
$$

(iii) Let $\sum_{\alpha \in \mathbb{N}^{n}} h_{\alpha} z^{\alpha}$ be the GS-ae of $f^{*}$, where $h_{\alpha} \in \mathscr{A}(U, E)$. Proposition 4.3 implies that

$$
\lim _{\substack{z \rightarrow 0 \\ z \in T}} \frac{D^{\alpha} f^{*}(z)}{\alpha !}=h_{\alpha}
$$


from this and (3), we conclude that

$$
h_{\alpha}(\omega)=\lim _{\substack{z \rightarrow 0 \\ z \in T}} \frac{\left(D^{\alpha} f^{*}(z)\right)(\omega)}{\alpha !}=\lim _{\substack{z \rightarrow 0 \\ z \in T}} \frac{D^{(\alpha, 0)} f(z, \omega)}{\alpha !}=f_{(\alpha, 0)}(\omega)
$$

for every $\omega \in U$. Put $\left(D^{(0, r)} f\right)^{*} \sim \sum_{\alpha \in \mathbb{N}^{n}} g_{\alpha} z^{\alpha}$. If $\mathscr{H}(U, E)$ is given the compactopen topology, the functions $f^{*}$ and $\left(D^{(0 . \gamma)} f\right)^{*}$ admit, as elements of $\mathscr{A}(S, \mathscr{H}(U, E))$, the same expansions $\sum f_{(\alpha, 0)} z^{\alpha}$ and $\sum g_{\alpha} z^{\alpha}$, respectively. So, for every $p \in \hat{E}$, $T \ll S, v \in \mathbb{N}$ and $K \subset U$ compact, there exists a constant $C(K)>0$ (depending on $p, T, v$ and $K$ ) such that for all $z \in T$,

$$
\sup \left\{p\left(f^{*}(z)(\omega)-\sum_{j=0}^{\nu} \sum_{|\boldsymbol{\alpha}|=j} f_{(\boldsymbol{\alpha}, \boldsymbol{0})}(\boldsymbol{\omega}) z^{\alpha}\right): \omega \in K\right\} \leq C(K)\|z\|^{\nu+1} .
$$

Take a compact subset $H$ of $U$; for $\omega \in H$ there exists $\delta_{\omega}>0$ such that $\bar{D}_{\delta_{\omega}}(\omega) \subset U$, and we can choose $\omega_{1}, \omega_{2}, \ldots, \omega_{k} \in H$ so that $H \subset \bigcup_{j=1}^{k} \bar{D}_{\delta_{\omega_{j} / 2}}(\omega)$. If $\omega=$ $\left(\omega_{1}, \ldots, \omega_{m}\right) \in H$, for some $s \in\{1,2, \ldots, k\}$ and for all $z \in S$ we can write

$$
\begin{aligned}
& D^{(0, \gamma)} f(z, \omega)-\sum_{j=0}^{\nu} \sum_{|\boldsymbol{\alpha}|=j} D^{\gamma} f_{(\boldsymbol{\alpha}, \mathbf{0})}(\boldsymbol{\omega}) z^{\alpha} \\
& =\frac{\gamma !}{(2 \pi i)^{m}} \int_{\partial_{0} D_{\delta_{\omega,}}(\omega,)} \frac{f(z, t)-\sum_{j=0}^{\nu} \sum_{|\boldsymbol{\alpha}|=j} f_{(\alpha, 0)}(t) z^{\alpha}}{(t-\omega)^{\gamma+1}} d t .
\end{aligned}
$$

If $t=\left(t_{1}, \ldots, t_{m}\right) \in \partial_{0} D_{\delta_{\omega_{s}}}\left(\omega_{s}\right)$ and $\omega_{s}=\left(\omega_{s 1}, \ldots, \omega_{s m}\right)$, we have

$$
\left|t_{q}-\omega_{q}\right| \geq\left|t_{q}-\omega_{s q}\right|-\left|\omega_{s q}-\omega_{q}\right| \geq \delta_{\omega_{s}} / 2
$$

hence, for $z \in T$ we get

$$
\begin{aligned}
& p\left(D^{(0, \gamma)} f(z, \omega)-\sum_{j=0}^{\nu} \sum_{|\alpha|=j} D^{\gamma} f_{(\alpha, 0)}(\omega) z^{\alpha}\right) \\
& \leq \frac{\gamma !}{(2 \pi)^{m}} \frac{2^{|\gamma|+m}}{\left(\delta_{\omega_{s}}\right)^{|\gamma|+m}} \int_{\partial_{0} D_{\delta_{\omega_{1}}(\omega,)}} p\left(f(z, t)-\sum_{j=0}^{\nu} \sum_{|\boldsymbol{\alpha}|=j} f_{(\boldsymbol{\alpha}, 0)}(t) z^{\alpha}\right)|d t| \\
& \leq \boldsymbol{\gamma} ! 2^{|\boldsymbol{y}|+m} \frac{C\left(\bar{D}_{\delta_{\omega_{s}}}\left(\omega_{s}\right)\right)}{\left(\delta_{\omega_{s}}\right)^{|\boldsymbol{\gamma}|}}\|z\|^{r+1} .
\end{aligned}
$$

Putting

$$
M_{H}=\sup _{1 \leq j \leq k} \frac{C\left(\bar{D}_{\delta_{\omega_{j}}}\left(\omega_{j}\right)\right)}{\left(\delta_{\omega_{j}}\right)^{|r|}}
$$


we conclude that for every $z \in T$ and $\omega \in H$,

$$
p\left(D^{(\boldsymbol{0}, \boldsymbol{\gamma})} f(\boldsymbol{z}, \boldsymbol{\omega})-\sum_{j=0}^{\nu} \sum_{|\boldsymbol{\alpha}|=j} D^{\gamma} f_{(\boldsymbol{\alpha}, 0)}(\boldsymbol{\omega}) z^{\alpha}\right) \leq \boldsymbol{\gamma} ! 2^{|\gamma|+m} M_{H}\|z\|^{v+1},
$$

so that $\left(D^{(0, \gamma)} f\right)^{*}: S \rightarrow \mathscr{H}(U, E)$ admits $\sum_{\alpha \in \mathbb{N}^{n}} D^{\gamma} f_{(\alpha, 0)} z^{\alpha}$ as GS-ae. Since such an expansion is unique, $D^{\gamma} f_{(\boldsymbol{\alpha}, \mathbf{0})}=g_{\boldsymbol{\alpha}}$ for every $\boldsymbol{\alpha} \in \mathbb{N}^{n}$.

\section{References}

[GS] R. Gérard and Y. Sibuya, 'Étude de certains systèmes de Pfaff avec singularités', in: Lecture Notes in Math. 172 (Springer, Berlin, 1979) pp. 131-288.

[Ha] Y. Haraoka, 'Theorems of Sibuya-Malgrange type for Gevrey functions of several variables', Funkcial. Ekvac. 32 (1989), 365-388.

[He] J. A. Hernández, Desarrollos asintóticos en polisectores. Problemas de existencia y unicidad (Ph. D. Thesis, University of Valladolid, 1994).

[HS] J. A. Hernández and J. Sanz, 'Constructive Borel-Ritt interpolation results for functions of several variables', Asymptotic Anal. 24 (2000), 167-182.

[M1] H. Majima, 'Analogues of Cartan's decomposition theorem in asymptotic analysis', Funkcial. Ekvac. 26 (1983), 131-154.

[M2] H. Majima, Asymptotic analysis for integrable connections with irregular singular points, Lecture Notes in Math. 1075 (Springer, Berlin, 1984).

[P] H. Poincaré, 'Sur les intégrales des équations linéaires', Acta Math. 8 (1886), 295-344.

[R] W. Rudin, Análisis real y complejo (McGraw-Hill/Interamericana, Madrid, 1988), translation into Spanish of Real and complex analysis, 3rd Edition (McGraw-Hill, New York, 1987).

[SG] J. Sanz and F. Galindo, 'On strongly asymptotically developable functions and the Borel-Ritt theorem', Studia Math. 133 (1999), 231-248.

[W] W. Wasow, Asymptotic expansions for ordinary differential equations (John Wiley and Sons, New York, 1965).

[Z] M. A. Zurro, 'A new Taylor type formula and $\mathscr{C}^{\infty}$ extensions for asymptotically developable functions', Studia Math. 123 (1997), 151-163.

Depto. de Análisis Matemático

Facultad de Ciencias

Universidad de Valladolid

c/ Prado de la Magdalena s/n

47005 Valladolid

Spain

e-mail: jaisla@am.uva.es

e-mail: jsanzg@am.uva.es 